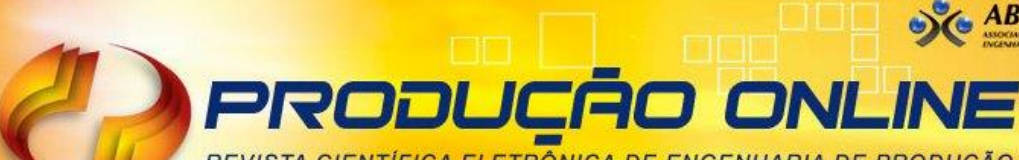 \\ REVISTA CIENTIFICA ELETRÔNICA DE ENGENHARIA DE PRODUÇÃO \\ ISSN 1676-1901
}

\section{EXPECTATIVAS E PERCEPÇÕES DA QUALIDADE DOS SERVIÇOS DE TRANSPORTE PRIVADO DE PASSAGEIROS SOLICITADO POR APLICATIVOS: EVIDÊNCIAS DO BRASIL}

\section{EXPECTATIONS AND PERCEPTIONS OF PRIVATE PASSENGER TRANSPORT SERVICES QUALITY REQUESTED BY APP: EVIDENCE FROM BRAZIL}

\author{
Lucas Rodrigues Deliberador*E-mail: deliberadorlucas@gmail.com \\ Amanda Oliveira Fontenelle*E-mail: amandaoliveirafontenelle@gmail.com \\ Fabiane Letícia LizarelliE-mail: fabiane@dep.ufscar.br \\ Mário Otávio Batalha*E-mail: dmob@ufscar.br \\ Juliana Keiko Sagawa*E-mail: juliana@dep.ufscar.br \\ *Universidade Federal de São Carlos (UFSCar), São Carlos, SP
}

\begin{abstract}
Resumo: O serviço de transporte privado solicitado por aplicativos de mobilidade surgiu em um contexto de ineficiência dos serviços de transporte público e da falta de universalidade na abrangência dos táxis, impulsionado também pela utilização cada vez mais crescente da tecnologia digital. Considerando que, no Brasil, pesquisas demonstram que os consumidores utilizam transportes por aplicativos de maneira crescente, o presente estudo objetiva verificar qual é a percepção dos passageiros em relação aos atributos envolvidos na satisfação com o serviço de transporte terrestre de passageiros oferecido por aplicativos. Os atributos foram definidos a partir de uma revisão de literatura e foram aplicados questionários on-line à 417 respondentes para averiguar como esses aspectos são avaliados pelos consumidores. Os resultados obtidos foram tratados por análise estatística da escala SERVQUAL, que possibilitou identificar a existência de uma lacuna negativa entre a expectativa dos consumidores e o desempenho real do serviço prestado para todos os itens avaliados. A partir desses resultados, as empresas de aplicativos de transporte podem trabalhar em melhorar a experiência que desejam proporcionar em sua prestação de serviço, na concepção de seu preço e de suas promoções, entre outros aspectos.
\end{abstract}

Palavras-chaves: Transporte por aplicativo. Qualidade em serviços. Percepção do consumidor. SERVQUAL. Brasil.

\begin{abstract}
The private transport service requested by mobility apps has emerged in a context of inefficient public transportation services and a lack of universality in taxi services, also driven by the increasing use of digital technology. Considering that there is a growth in the use of transportation by applications in Brazil, the present study aims to verify the passenger's perception regarding the satisfaction's atributes with the transportation service offered by apps. Attributes were defined from a preliminary literature review and 417 online questionnaires were applied to ascertain how these aspects are evaluated by consumers. The results were treated by statistical analysis of the SERVQUAL scale, which made it possible to identify the existence of a negative gap between consumers' expectations and the actual performance of the service provided. From these results, app transportation companies can work on improving the experience they want to provide in their service, pricing design and promotions, among other things.
\end{abstract}

Keywords: App transportation. Quality service. Customer perception. SERVQUAL. Brazil. 


\section{INTRODUÇÃO}

Com o desenvolvimento das tecnologias e a exigência de maior rapidez e abrangência no atendimento das necessidades dos consumidores, uma nova lógica de mercado surgiu, trazendo a necessidade do desenvolvimento de novos mecanismos e adequações às organizações no que diz respeito a produtos e serviços. Assim, o serviço de transporte solicitado por aplicativos de mobilidade foi um dos tipos de serviço que surgiu nesse contexto de mudanças e impulsionado também pela utilização cada vez mais crescente da tecnologia digital.

A partir de aplicativos para smartphones e outros dispositivos, é possível fornecer serviços para clientes que solicitam transporte. Essa alternativa surgiu a partir da observação na ineficiência dos serviços de transporte público e da falta de universalidade na abrangência dos táxis, que seriam, em teoria, os principais concorrentes desse serviço (ZHANG et al., 2016).

Segundo pesquisa financiada pelo SPC Brasil em parceria com a Confederação Nacional de Dirigentes Lojistas (CNDL) em 2017, em um universo que considera todos os meios de transporte, $49,7 \%$ dos entrevistados afirmaram que preferem fazer compras utilizando serviços de transporte privados, por serem mais baratos, de mais fácil acesso e mais cômodos para suas necessidades. Além disso, se considerado nessa mesma amostra apenas os respondentes que utilizam táxis ou os demais transportes por aplicativos, $64,6 \%$ afirmaram utilizar mais os transportes por aplicativos do que os tradicionais táxis.

Este contexto demonstra a crescente movimentação de passageiros por essa modalidade de transporte e consequentemente estimula a seguinte reflexão: qual é a percepção dos passageiros do serviço de transporte solicitado por aplicativos de mobilidade no Brasil? Para captar essa percepção, é necessário compreender qual é a visão dos consumidores a respeito dos atributos existentes nesse tipo de serviço. Quando o prestador de serviço compreende de que forma o serviço é avaliado pelo consumidor, é possível administrar os atributos e conduzí-los em direção à satisfação do cliente (RODRIGUES, 2001).

Desta forma, o presente estudo objetiva verificar qual é a percepção dos passageiros em relação aos atributos envolvidos na satisfação com o serviço de transporte terrestre de passageiros oferecido por aplicativos no Brasil. Entende-se 
que a qualidade do serviço prestado não só influencia a satisfação do consumidor, mas também é determinante para a escolha do consumidor entre um aplicativo em prol de outro, já que coexistem várias alternativas de aplicativos de transporte atualmente no mercado. Além disso, devido à rápida expansão desses serviços, convém questionar-se sobre quais as características apreciadas pelos usuários e qual a avaliação considerada por eles a respeito desses serviços de transporte (COELHO et al., 2017).

Para identificar quais os atributos que serão avaliados, foram selecionados e discutidos alguns dos principais aspectos existentes relacionados ao serviço de transporte terrestre de passageiros por aplicativos. Foram aplicados questionários para averiguar como esses aspectos são avaliados pelos consumidores. Os resultados obtidos foram tratados por análise estatística da escala SERVQUAL proposta por Parasuraman et al. $(1985,1988,1991)$. A partir disso, foi possível fazer uma análise geral dos atributos na visão dos consumidores, traçando um paralelo entre a expectativa dos clientes e as percepções captadas em suas experiências na utilização desses serviços.

\section{TRANSPORTE INDIVIDUAL DE PASSAGEIROS POR APLICATIVOS}

No âmbito da mobilidade urbana, o transporte de passageiros por aplicativo traz um novo formato de consumo, baseado em plataformas tecnológicas, que possibilitam a interação entre motoristas e usuários interessados em utilizar o serviço. São conhecidos como aplicativos de e-hailing (EHA) aqueles que gerenciam o processo de encomendar o serviço de transporte de um carro, limusine, ou qualquer outro transporte de passageiros através de dispositivo móvel (JOIA; ALTIERI, 2017).

Observou-se que o crescimento da utilização desses EHA no Brasil foi derivado de uma demanda crescente e uma oferta insuficiente de serviço privado de transporte, associada ao aumento da utilização de smartphones. Essa tecnologia dos EHA pode ser considerada como disruptiva, visto que afetou diretamente o modelo tradicional de táxis, oferecendo diferentes valores e facilidades anteriormente não associados a este tipo de serviço (MARÔCCO et al., 2014).

Tratando-se especificamente sobre o serviço de transporte privado feito por 
veículos automotivos, os motoristas que estão cadastrados como parceiros são solicitados para atender a chamados dos passageiros. Antes mesmo dos clientes solicitarem o serviço, conseguem saber qual o valor previsto a ser cobrado e qual o tempo previsto para o veículo chegar ao seu encontro. A fixação dos preços é totalmente controlada pelas plataformas e os motoristas não exercem nenhuma influência sobre tal questão. Além disso, alguns aplicativos apresentam o preço dinâmico, que pode ser alterado momentaneamente de acordo com os algoritmos que funcionam como uma ferramenta de controle de demanda (TEODORO; DA SILVA; ANTONIETA, 2017).

Essas facilidades fazem parte de aspectos intangíveis oferecidos na experiência de utilização dos aplicativos, bem como outras informações relevantes, tais como: quais são os dados do veículo e do motorista, quais são as avaliações de outros usuários para o motorista, qual é o melhor percurso a ser feito durante o trajeto e qual é a estimativa de tempo para chegar ao destino. Além disso, em algumas plataformas é possível conversar com o motorista por meio de um canal de comunicação virtual dentro do aplicativo, que visa melhorar a comunicabilidade entre passageiro e motorista e facilita não apenas o encontro, mas também a acessibilidade do passageiro, caso necessário.

Todas as funcionalidades existentes nos aplicativos são atrativas não apenas para os consumidores, mas também para os motoristas parceiros. Em um estudo realizado nos Estados Unidos voltado para o aplicativo de transporte mais utilizado no país, observou-se que a oferta flexível de trabalho proporcionada pelos aplicativos aos condutores pode atrair trabalhadores que fornecem mão-de-obra para os setores em geral (HALL; KRUEGER, 2018). A facilidade em se cadastrar e exercer a atividade sem uma obrigatoriedade de horários é interessante para um perfil de pessoas desempregadas, bem como para aqueles que desejam complementar suas rendas ou que não gostariam de cumprir um horário rígido de trabalho.

O outro aspecto atraente e que justifica o crescimento da oferta desse tipo de serviço é que os motoristas de aplicativos que se dedicaram integralmente ganham tanto quanto, ou mais, que motoristas de táxi pesquisados (HALL; KRUEGER, 2018). Contudo, alguns trabalhos demonstram que a perspectiva de flexibilidade deve ser avaliada com cautela, visto que, existem indefinições na regulamentação Revista Produção Online. Florianópolis, SC, v. 20, n. 4, p. 1384-1404, 2020. 
desse tipo de serviço em vários países, que podem comprometer a qualidade de vida do motorista, principalmente no que tange às longas horas de prestação de serviço (TEODORO; DA SILVA; ANTONIETA, 2017).

Da mesma forma, a flexibilidade na prestação desse serviço também pode comprometer aspectos relacionados à qualidade do serviço. As pessoas interessadas em prestar esse serviço de transporte por aplicativo são cadastradas como motoristas parceiros e não há obrigatoriamente um processo de seleção. Desta forma, é possível que o serviço prestado seja insatisfatório pela visão do cliente, já que o aplicativo não garante que atributos dependentes da habilidade do motorista em lidar com pessoas ou em resolver possíveis problemas sejam satisfatórios. Os passageiros esperam motoristas bem apresentados, educados e simpáticos (CIRNE; BARROS, 2015), mas as empresas, ao contratarem sem um processo de seleção de pessoas, não conseguem ser necessariamente assertivas no credenciamento dos prestadores de serviços.

Outros atributos também podem ser vistos como de responsabilidade direta pela gestão do aplicativo, por exemplo, os preços justos de acordo com a distância e o destino. Em relação a esses atributos, os motoristas, na maioria das vezes, não conseguem interferir ou ajudar. Caso os atributos que estão sob responsabilidade da gestão da empresa não sejam bem avaliados pelos clientes, pode-se gerar resistência na utilização das plataformas. Além dos atributos já mencionados, outros podem depender de uma gestão conjunta entre aplicativo e motorista, como, o caso da resolução rápida de eventuais problemas dos clientes com o uso aplicativo.

Além disso, alguns atributos tangíveis podem ser esperados de um serviço de transporte privado de passageiros, como oferecer carros novos, limpos e dentro de certos requisitos determinados pela empresa (CIRNE; BARROS, 2015). Alguns aplicativos implantaram as avaliações dos motoristas para facilitar a gestão da reputação e assim credenciá-los ou descredenciá-los de acordo com a avaliação da conduta. Contudo, um consumidor que possui uma experiência ruim, avalia sob a perspectiva da sua própria tolerância a utilização novamente ou não do serviço. Isso pode vir a ser um ponto crítico na escolha entre um aplicativo em prol de outro e também na sustentabilidade das operações, já que a empresa não se sustenta financeiramente pela mera disponibilização do aplicativo, mas pelas corridas efetivamente contratadas pelos passageiros (T. MÁXIMO; D'AFONSECA; 
ANTONIETA, 2017).

Por tratar-se de um tema recente, a literatura carece de estudos sobre as características do serviço de transporte de passageiros por aplicativos (COELHO et al., 2017), no que se refere a atributos de acordo com a expectativa do consumidor. Entretanto, diante desse novo paradigma do mercado, torna-se necessário estudar como os usuários percebem e avaliam a qualidade desse serviço, considerando suas expectativas em contrapartida à percepção a respeito do serviço usufruído. $\mathrm{Na}$ próxima seção serão indicados os atributos investigados na pesquisa de acordo com Parasuraman et al. (1985, 1988, 1991) e o método aplicado para verificar a expectativa e a percepção captadas.

\section{QUALIDADE EM SERVIÇOS E SERVQUAL}

A qualidade do serviço é considerada um determinante crítico da competitividade. A atenção à qualidade do serviço pode ajudar uma organização a se diferenciar de outras organizações e, através dela, obter uma vantagem competitiva duradoura. Como um serviço é considerado intangível, o comprador não pode julgar a sua qualidade ou valor antes da compra e do consumo. A qualidade percebida está ao longo de um continuum. Dessa forma, a qualidade inaceitável está em uma ponta desse continuum, enquanto que a qualidade aceitável está no outro extremo. Os pontos intermediários representam diferentes gradações de qualidade (GHOBADIAN; SPELLER; JONES, 1994). A qualidade em serviços pode ser considerada como uma medida que objetiva verificar se o serviço oferecido atende às expectativas do consumidor (PARASURAMAN et al., 1985, 1988, 1991).

Diante desse contexto, a escala SERVQUAL foi introduzida e refinada por Parasuraman et al. (1985, 1988, 1991). Usando a escala SERVQUAL, os dados são coletados por meio de um questionário que aborda aspectos de expectativa e percepção (PRAKASH, 2019). Apesar de ser criticado em aspectos metodológicos e psicométricos (CRONIN; TAYLOR, 1992), o SERVQUAL tem sido amplamente utilizado (PRAKASH, 2019). O questionário é segmentado em 22 perguntas, agrupadas em cinco dimensões da qualidade em serviços, como podem ser descritas a seguir (PARASURAMAN et al., 1985, 1988, 1991):

(1) Confiabilidade: capacidade de executar serviços com precisão; 
(2) Tangibilidade: instalações físicas, pessoal, equipamento, construção, aparência, etc.;

(3) Responsividade: vontade de ajudar e responder às necessidades do cliente;

(4) Empatia: atenção, carinho e serviço individual são dados ao cliente;

(5) Segurança: o grau de conhecimento e cortesia dos funcionários de uma empresa; sua capacidade de inspirar confiança entre os clientes.

Em uma análise SERVQUAL, é calculada a diferença entre a qualidade percebida, ou percepção $(\mathrm{P})$, e a qualidade esperada, ou expectativa do consumidor (E). A diferença entre a percepção e a expectativa $(P-E)$ possibilita compreender o que é realmente observado e o que representaria um serviço ideal. Em alguns estudos que adotaram este método de análise, podem ser encontradas diferenças negativas $(P<E)$, indicando que existiu uma inadequação em atender às expectativas dos clientes.

Diversas aplicações da SERVQUAL foram realizadas em diferentes áreas, como na educação (ABDULLAH, 2006; BROCHADO, 2009; YUSOFF; MCLEAY; WOODRUFFE-BURTON, 2015; SILVA et al., 2019) saúde (RAMEZ, 2012; AKDERE; TOP; TEKINGÜNDÜZ, 2018), hospitalidade (SALAZAR; COSTA; RITA, 2010; UNUVAR; KAYA, 2017), bancos (NEWMAN, 2001; RAVICHANDRAN et al., 2010) etc. Este trabalho possui como objeto de estudo o setor de transporte. Alguns estudos, como é o caso de Santana et al. (2018) e Sam, Hamidu e Daniels (2018), já realizaram pesquisas na área de transporte. Santana et al. (2018) utilizou-se do instrumento SERVQUAL para avaliar os serviços fornecidos pelas empresas brasileiras de aviação civil, considerando os voos domésticos. Sam, Hamidu e Daniels (2018) analisaram as expectativas e percepções de qualidade de serviço dos usuários do transporte coletivo em Gana.

Diante do contexto apresentado, apesar dos estudos mencionados e outros presentes na literatura englobando o serviço de transporte, verifica-se que ainda há uma deficiência substancial de aplicações na área de transporte individual de passageiros por aplicativos, com nenhuma tentativa prévia encontrada de apresentar uma estrutura para a medição da qualidade do serviço por meio de um procedimento SERVQUAL. Desse modo, ao preencher esta essa lacuna, este estudo pode ser de grande utilidade para as empresas prestadoras de serviço de transporte remunerado 
privado individual, especialmente para aquelas que desejam cumprir com os requisitos listados pelo método aqui discutido.

\section{PROCEDIMENTOS METODOLÓGICOS}

Esta seção apresenta os procedimentos metodológicos do estudo. A seção encontra-se dividida em: (1) objeto de estudo definição da amostra, (2) coleta de dados, e (3) análise dos dados.

\subsection{Objeto de estudo e definição da amostra}

O objeto de estudo desta pesquisa são os usuários do transporte de passageiros por aplicativos no Brasil. Este estudo objetiva verificar qual é a percepção dos consumidores acerca da qualidade dos serviços oferecidos pelo transporte individual de passageiros. A amostra analisada para a realização da pesquisa foi calculada considerando uma população infinita. Para este cálculo, um nível de $95 \%$ de significância foi considerado $\left(Z_{\alpha / 2}\right)$ e um erro amostral $(E)$ de $5 \%$. Desse modo, ao menos 385 indivíduos deveriam ser selecionados a participarem do estudo.

\subsection{Coleta de dados}

Os dados foram coletados em junho de 2019, e contemplou somente os consumidores brasileiros. Através de um questionário estruturado, foram coletadas informações que buscasse obter uma caracterização da amostra (seção 1) e informações que verificaram a percepção dos consumidores em relação à qualidade do serviço de transporte de passageiros por aplicativo (seção 2). A seção 2 do questionário foi elaborada através de uma escala Likert de sete pontos, em que 7 indica uma visão forte e positiva do respondente, 4 indica visão neutra e 1 indica uma forte visão negativa.

Respostas do tipo Likert são esquemas populares de pontuação de itens psicométricos que objetivam quantificar as opiniões das pessoas em diferentes questões (BISHOP; HERRON, 2015). O questionário foi desenvolvido e 
disponibilizado aos respondentes por meio da plataforma virtual Google Forms ® Dados referentes às informações pessoais dos respondentes não foram coletados, preservando a identidade dos mesmos. As variáveis presentes no questionário foram baseadas/adaptadas nos estudos de Parasuraman et al. (1985, 1988, 1991).

\subsection{Análise dos dados}

Os dados foram analisados através do software Statistical Package for the Social Sciences (SPSS®). Inicialmente, foi verificada a confiabilidade dos resultados do questionário de acordo com o alfa de Cronbach ( $\alpha$ ). Quanto maior o valor do $\alpha$, que varia de 0 a 1, maior é a confiabilidade das dimensões verificadas (HAIR et al., 1998; TONGLET et al., 2004). Valores de a maiores que 0,7 já podem ser considerados confiáveis (HAIRS et al., 1998). Posteriormente, foi realizada uma análise de estatística descritiva para apresentar os valores de medidas de tendências centrais, como: média, mediana, desvio padrão e valores de máximo e mínimo. Por meio da escala SERVQUAL, foram verificadas as lacunas existentes entre as percepções e expectativas dos consumidores do transporte de passageiros por aplicativos. Para corroborar com a análise, foi realizado o teste $U$ de MannWhitney para determinar se as lacunas encontradas eram significativas. Neste estudo, foram consideradas lacunas significativamente estatísticas as que apresentaram $p \leq 0,05$. Finalmente, foi efetuada a análise de quartis para identificar quais são as variáveis com prioridade crítica, alta, moderada e baixa.

\section{RESULTADOS E DISCUSSÕES}

O questionário foi ajustado para avaliar a concordância dos entrevistados acerca das dimensões da qualidade de serviço: confiabilidade, tangibilidade, responsividade, segurança e empatia. Os 22 itens propostos por Parasuraman et al. (1988) foram selecionados para capturar essas cinco dimensões da qualidade no serviço de transporte individual de passageiros por aplicativo (Tabela 1). Para um melhor entendimento dos atributos, a Tabela 1 foi segmentada em duas partes, indicando a responsabilidade como sendo do motorista, do aplicativo ou de ambos. Dessa maneira, foram medidas as expectativas $(E)$ e as percepções $(P)$ dos 
consumidores. O questionário também incluiu perguntas sobre os dados demográficos dos respondentes (Tabela 2).

Tabela 1 - Itens para avaliação das expectativas e percepções dos passageiros de transporte por aplicativos

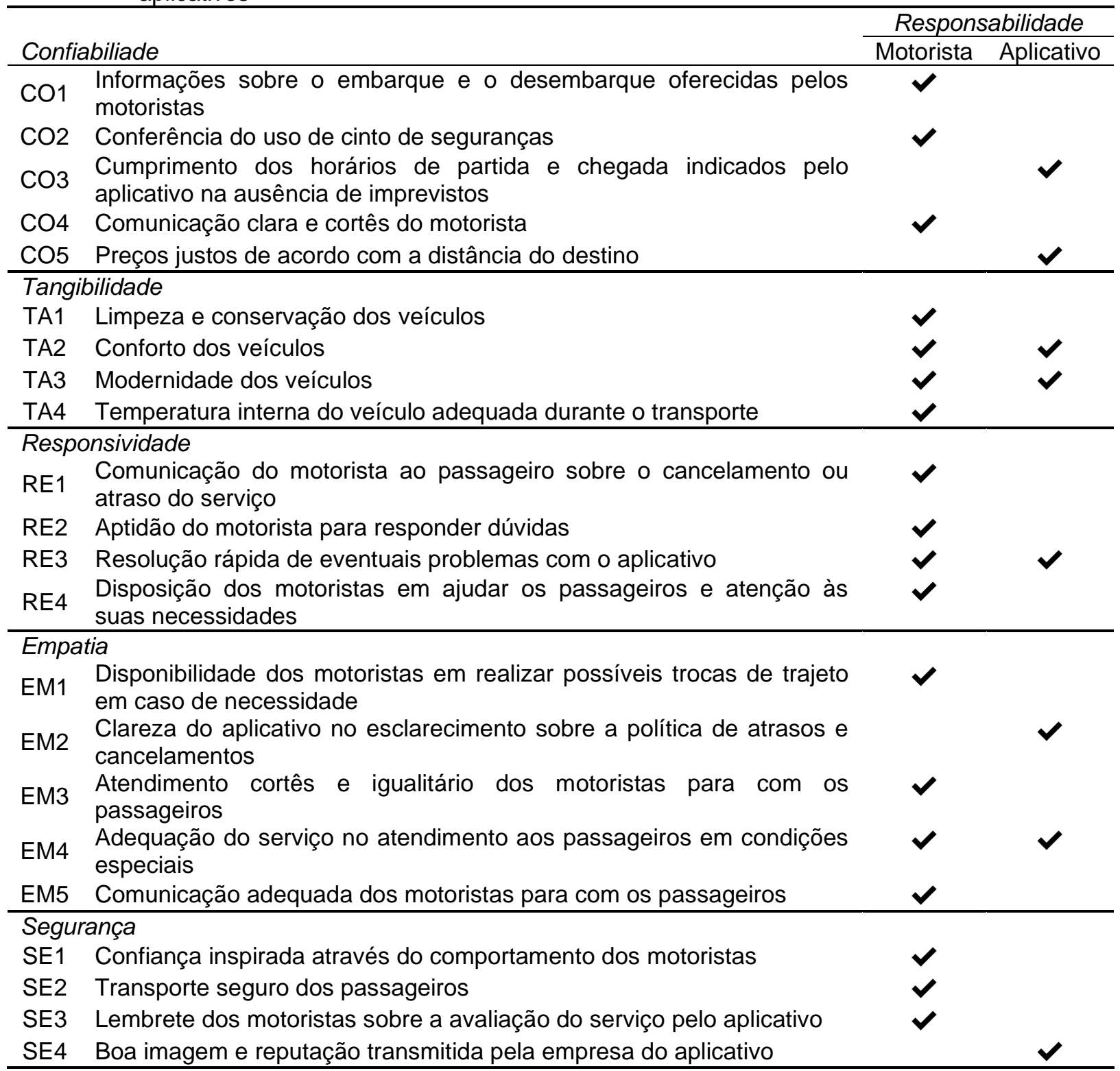

Fonte: Adaptado de Parasuraman et al. (1988).

Os dados demográficos dos respondentes são apresentados na Tabela 2. 417 consumidores participaram da pesquisa, sendo que $72,4 \%$ eram do sexo feminino. Dos entrevistados, 60,7\% possuíam entre 25 e 35 anos de idade. Além disso, mais de $72 \%$ dos entrevistados possuíam curso superior completo. É importante ressaltar que o questionário foi disponibilizado em uma plataforma online, o que é algo crescente e já predomina no domínio das pesquisas de satisfação dos

Revista Produção Online. Florianópolis, SC, v. 20, n. 4, p. 1384-1404, 2020. 
consumidores. Entretanto, é relevante reconhecer que existe uma tendência da amostra ser formada por participantes mais jovens e com maior nível educacional do que a média brasileira. Desse modo, os resultados aqui apresentados devem ser relativizados sob a perspectiva desta constatação.

Tabela 2 - Dados demográficos dos respondentes

\begin{tabular}{lcc}
\hline Variável & Frequência $(\mathrm{n})$ & Porcentagem $(\%)$ \\
\hline Sexo & 302 & 72,4 \\
\hline Feminino & 115 & 27,6 \\
Masculino & & 1,4 \\
\hline Faixa etária & 6 & 16,1 \\
\hline 18 anos & 67 & 60,7 \\
De 19 a 24 anos & 253 & 15,3 \\
De 25 a 35 anos & 64 & 6,5 \\
De 36 a 50 anos & 27 & 0 \\
A partir de 51 anos & & 0 \\
\hline Escolaridade & 0 & 1 \\
\hline Não frequentou a escola & 0 & 12,5 \\
Ensino fundamental incompleto & 4 & 4,6 \\
Ensino fundamental completo & 0 & 16,3 \\
Ensino médio incompleto & 19 & 72,2 \\
Ensino médio completo & 68 & 100 \\
Ensino superior incompleto & 326 & \\
Ensino superior completo & 417 & \\
\hline Total & & \\
\hline
\end{tabular}

Fonte: elaborado pelos autores.

Neste estudo, os valores do alfa de Cronbach foram utilizados para determinar a confiabilidade das seções do questionário que avaliaram as expectativas e percepções sobre 0 transporte individual de passageiros por aplicativos. Os valores do alfa de Cronbach dos constructos foram de 0,833 para as variáveis relacionadas às expectativas, e de 0,957 para as variáveis relacionadas à percepção, o que é considerado muito bom e excelente, conforme indicado pela literatura (HAIR et al., 2005).

A Tabela 3 apresenta os valores das medidas centrais, como média $(\bar{x})$, mediana, desvio padrão da amostra (s), dos resultados em relação às variáveis individuais das cinco dimensões para expectativa $(E)$ e percepção (P). Adicionalmente as medidas centrais, são apresentados a frequência de respostas e os valores máximo e mínimo da distribuição de frequência, apoiando a análise dos dados. Os valores de máximo e mínimo demonstram que para o se refere as percepções, houveram consumidores com percepção extremamente positivas, como também respostas com percepção consideradas extremamente negativas. No caso das expectativas, houveram respostas que demonstraram que para todos os itens Revista Produção Online. Florianópolis, SC, v. 20, n. 4, p. 1384-1404, 2020. 
investigados, existiam avaliações do maior nível possível. Porém, verificou-se através dos valores de mínimo, que um dos itens avaliados, referente ao esclarecimento sobre a política de atrasos e cancelamentos (EM2), apresentou expectativa mínima de valor 4, em uma escala de 1 a 7 , considerando os 417 respondentes. 
Tabela 3 - Medidas de tendências centrais do questionário

$$
\text { Expectativa }(E)
$$

\begin{tabular}{|c|c|c|c|c|c|c|c|c|c|c|c|c|c|c|c|c|c|c|c|c|c|c|c|c|}
\hline \multirow[t]{2}{*}{ D } & \multicolumn{7}{|c|}{ Frequência das Respostas } & \multirow{2}{*}{$\overline{\mathrm{x}}$} & \multirow{2}{*}{ Mediana } & \multirow{2}{*}{$s$} & \multirow{2}{*}{ Máx } & \multirow{2}{*}{ Mín } & \multicolumn{7}{|c|}{ Frequência das Respostas } & \multirow{2}{*}{$\overline{\mathrm{x}}$} & \multirow{2}{*}{ Mediana } & \multirow{2}{*}{$s$} & \multirow{2}{*}{ Máx } & \multirow{2}{*}{ Mín } \\
\hline & 1 & 2 & 3 & 4 & 5 & 6 & 7 & & & & & & 1 & 2 & 3 & 4 & 5 & 6 & 7 & & & & & \\
\hline \multicolumn{25}{|c|}{ Confiabiliade } \\
\hline $\mathrm{CO} 1$ & 7 & 9 & 13 & 50 & 69 & 62 & 207 & 5,83 & 6,00 & 1,471 & 7 & 1 & 20 & 28 & 49 & 76 & 97 & 41 & 86 & 4,65 & 5,00 & 1,820 & 7 & 1 \\
\hline $\mathrm{CO} 2$ & 11 & 6 & 9 & 23 & 37 & 43 & 288 & 6,24 & 7,00 & 1,426 & 7 & 1 & 130 & 46 & 50 & 48 & 31 & 24 & 88 & 3,55 & 3,00 & 2,319 & 7 & 1 \\
\hline $\mathrm{CO} 3$ & 9 & 1 & 6 & 20 & 27 & 43 & 311 & 6,42 & 7,00 & 1,238 & 7 & 1 & 20 & 21 & 30 & 57 & 70 & 85 & 134 & 5,22 & 6,00 & 1,758 & 7 & 1 \\
\hline $\mathrm{CO} 4$ & 1 & 1 & 2 & 6 & 13 & 38 & 356 & 6,76 & 7,00 & 0,715 & 7 & 1 & 10 & 7 & 32 & 70 & 95 & 86 & 117 & 5,30 & 5,00 & 1,501 & 7 & 1 \\
\hline $\mathrm{CO5}$ & 3 & 0 & 0 & 8 & 12 & 18 & 376 & 6,80 & 7,00 & 0,742 & 7 & 1 & 15 & 13 & 25 & 50 & 77 & 78 & 159 & 5,47 & 6,00 & 1,647 & 7 & 1 \\
\hline \multicolumn{25}{|c|}{ Tangibilidade } \\
\hline TA1 & 1 & 0 & 1 & 5 & 14 & 29 & 367 & 6,80 & 7,00 & 0,631 & 7 & 1 & 12 & 10 & 32 & 77 & 82 & 78 & 126 & 5,27 & 5,00 & 1,581 & 7 & 1 \\
\hline TA2 & 1 & 2 & 3 & 24 & 65 & 50 & 272 & 6,33 & 7,00 & 1,065 & 7 & 1 & 7 & 12 & 22 & 87 & 100 & 80 & 109 & 5,25 & 5,00 & 1,459 & 7 & 1 \\
\hline TA3 & 14 & 15 & 30 & 116 & 122 & 49 & 71 & 4,79 & 5,00 & 1,479 & 7 & 1 & 11 & 22 & 56 & 116 & 108 & 49 & 55 & 4,57 & 5,00 & 1,476 & 7 & 1 \\
\hline TA4 & 3 & 4 & 11 & 41 & 70 & 62 & 226 & 6,02 & 7,00 & 1,295 & 7 & 1 & 11 & 15 & 28 & 75 & 89 & 83 & 116 & 5,23 & 5,00 & 1,568 & 7 & 1 \\
\hline \multicolumn{25}{|c|}{ Responsividade } \\
\hline RE1 & 2 & 0 & 0 & 3 & 7 & 18 & 387 & 6,87 & 7,00 & 0,577 & 7 & 1 & 65 & 45 & 55 & 63 & 44 & 36 & 109 & 4,25 & 4,00 & 2,173 & 7 & 1 \\
\hline RE2 & 6 & 6 & 12 & 41 & 78 & 84 & 190 & 5,86 & 6,00 & 1,367 & 7 & 1 & 15 & 17 & 46 & 102 & 89 & 62 & 86 & 4,83 & 5,00 & 1,606 & 7 & 1 \\
\hline RE3 & 14 & 14 & 14 & 57 & 78 & 81 & 159 & 5,52 & 6,00 & 1,602 & 7 & 1 & 36 & 32 & 54 & 95 & 81 & 43 & 76 & 4,41 & 4,00 & 1,818 & 7 & 1 \\
\hline RE4 & 4 & 3 & 8 & 16 & 74 & 74 & 238 & 6,18 & 7,00 & 1,183 & 7 & 1 & 8 & 21 & 38 & 70 & 93 & 79 & 108 & 5,13 & 5,00 & 1,583 & 7 & 1 \\
\hline \multicolumn{25}{|c|}{ Empatia } \\
\hline EM1 & 6 & 2 & 8 & 34 & 57 & 81 & 229 & 6,10 & 7,00 & 1,271 & 7 & 1 & 16 & 15 & 28 & 48 & 80 & 101 & 120 & 5,35 & 6,00 & 1,639 & 7 & 1 \\
\hline EM2 & 0 & 0 & 0 & 3 & 10 & 22 & 382 & 6,88 & 7,00 & 0,446 & 7 & 4 & 46 & 39 & 42 & 62 & 63 & 54 & 111 & 4,59 & 5,00 & 2,048 & 7 & 1 \\
\hline EM3 & 1 & 0 & 1 & 4 & 6 & 20 & 385 & 6,87 & 7,00 & 0,538 & 7 & 1 & 13 & 17 & 36 & 64 & 82 & 89 & 116 & 5,20 & 5,00 & 1,630 & 7 & 1 \\
\hline EM4 & 2 & 0 & 3 & 8 & 15 & 31 & 358 & 6,74 & 7,00 & 0,782 & 7 & 1 & 45 & 48 & 52 & 91 & 51 & 34 & 96 & 4,30 & 4,00 & 1,999 & 7 & 1 \\
\hline EM5 & 1 & 0 & 1 & 4 & 17 & 37 & 357 & 6,78 & 7,00 & 0,643 & 7 & 1 & 3 & 18 & 39 & 75 & 86 & 87 & 109 & 5,21 & 5,00 & 1,508 & 7 & 1 \\
\hline \multicolumn{25}{|c|}{ Segurança } \\
\hline SE1 & 1 & 0 & 2 & 6 & 14 & 37 & 357 & 6,77 & 7,00 & 0,680 & 7 & 1 & 6 & 18 & 41 & 76 & 96 & 73 & 107 & 5,12 & 5,00 & 1,545 & 7 & 1 \\
\hline SE2 & 1 & 0 & 0 & 0 & 4 & 2 & 400 & 6,94 & 7,00 & 0,387 & 7 & 1 & 2 & 8 & 23 & 85 & 94 & 77 & 128 & 5,41 & 5,00 & 1,387 & 7 & 1 \\
\hline SE3 & 19 & 10 & 21 & 66 & 78 & 54 & 169 & 5,43 & 6,00 & 1,697 & 7 & 1 & 45 & 18 & 41 & 58 & 59 & 80 & 116 & 4,85 & 5,00 & 1,958 & 7 & 1 \\
\hline SE4 & 1 & 0 & 1 & 4 & 10 & 39 & 362 & 6,81 & 7,00 & 0,603 & 7 & 1 & 9 & 11 & 20 & 52 & 85 & 103 & 137 & 5,52 & 6,00 & 1,482 & 7 & 1 \\
\hline
\end{tabular}

Legenda: D - Dimensão.

Fonte: elaborado pelos autores. 
As lacunas $(\Delta)$ e os respectivos rankings são apresentados na Tabela 4. A maior lacuna $(-2,69)$ encontrada é atribuída ao segundo item da dimensão confiabilidade (CO2): conferência dos cintos de segurança por parte dos motoristas. Todos os 22 itens experimentaram algum tipo de lacuna negativa. As pontuações negativas indicam a existência de lacunas de qualidade de serviço, onde os entrevistados não estão tendo suas expectativas atendidas pelo desempenho real do serviço. O item com a menor lacuna, e que, portanto, esteve próximo de atender as expectativas dos consumidores, foi o terceiro da dimensão tangibilidade (TA3): modernidade dos veículos. A Figura 1 ilustra através do gráfico de radar as lacunas dos 22 itens.

Como os dados da amostra são não-paramétricos, o teste $U$ de MannWhitney foi considerado para determinar estatisticamente se havia diferenças significativas entre as expectativas e as percepções da qualidade do serviço de transporte de passageiros por aplicativos. O teste $U$ de Mann-Whitney confirmou que para os 22 itens das cinco dimensões, os consumidores tiveram percepções significativamente mais baixas do que as suas expectativas $(Z=1,96$ e $p<0,05$ para todas os itens das cinco dimensões). A maior diferença foi do item RE1 da dimensão de responsividade, seguida dos itens EM2, SE2, EM4 e EM3, respectivamente.

Tabela 4 - Resultados das lacunas e teste U de Mann-Whitney

\begin{tabular}{|c|c|c|c|c|c|c|c|}
\hline Dimensões & Variáveis & $\begin{array}{c}\Delta \\
(P-E)\end{array}$ & $\Delta$ Ranking & $\begin{array}{c}\text { Mann- } \\
\text { Whitney U }\end{array}$ & Z & Z Ranking & $\begin{array}{c}\text { Asymp. Sig. (2 } \\
\text { tailed) }\end{array}$ \\
\hline \multirow{5}{*}{ Confiabilidade } & $\mathrm{CO} 1$ & $-1,18$ & 14 & 54078,000 & $-9,755$ & 16 & 0,000 \\
\hline & $\mathrm{CO} 2$ & $-2,69$ & 1 & 32930,500 & $-16,357$ & 10 & 0,000 \\
\hline & $\mathrm{CO} 3$ & $-1,20$ & 13 & 47850,000 & $-12,248$ & 13 & 0,000 \\
\hline & $\mathrm{CO} 4$ & $-1,46$ & 10 & 33744,000 & $-16,98$ & 9 & 0,000 \\
\hline & CO5 & $-1,33$ & 11 & 40702,500 & $-15,528$ & 12 & 0,000 \\
\hline \multirow{4}{*}{ Tangibilidade } & TA1 & $-1,53$ & 8 & 33748,500 & $-17,219$ & 8 & 0,000 \\
\hline & TA2 & $-1,08$ & 16 & 48171,500 & $-11,81$ & 14 & 0,000 \\
\hline & TA3 & $-0,22$ & 22 & 78810,000 & $-2,398$ & 22 & 0,016 \\
\hline & TA4 & $-0,79$ & 19 & 60239,000 & $-8,021$ & 19 & 0,000 \\
\hline \multirow{4}{*}{ Responsividade } & RE1 & $-2,62$ & 2 & 25977,000 & $-19,748$ & 1 & 0,000 \\
\hline & RE2 & $-1,03$ & 18 & 53983,000 & $-9,748$ & 17 & 0,000 \\
\hline & RE3 & $-1,11$ & 15 & 55692,000 & $-9,168$ & 18 & 0,000 \\
\hline & RE4 & $-1,05$ & 17 & 51885,500 & $-10,548$ & 15 & 0,000 \\
\hline \multirow{5}{*}{ Empatia } & EM1 & $-0,75$ & 20 & 62273,000 & $-7,456$ & 20 & 0,000 \\
\hline & EM2 & $-2,29$ & 4 & 26979,000 & $-19,381$ & 2 & 0,000 \\
\hline & EM3 & $-1,67$ & 5 & 29071,500 & $-18,848$ & 5 & 0,000 \\
\hline & EM4 & $-2,44$ & 3 & 26514,500 & $-19,007$ & 4 & 0,000 \\
\hline & EM5 & $-1,57$ & 7 & 31232,000 & $-17,693$ & 7 & 0,000 \\
\hline \multirow{4}{*}{ Segurança } & SE1 & $-1,65$ & 6 & 30644,000 & $-17,851$ & 6 & 0,000 \\
\hline & SE2 & $-1,53$ & 9 & 29022,000 & $-19,327$ & 3 & 0,000 \\
\hline & SE3 & $-0,58$ & 21 & 72688,000 & $-4,211$ & 21 & 0,000 \\
\hline & SE4 & $-1,29$ & 12 & 37177,000 & $-16,209$ & 11 & 0,000 \\
\hline
\end{tabular}

Fonte: elaborado pelos autores. 
Figura 1 - Lacunas entre a percepção e a expectativa dos consumidores

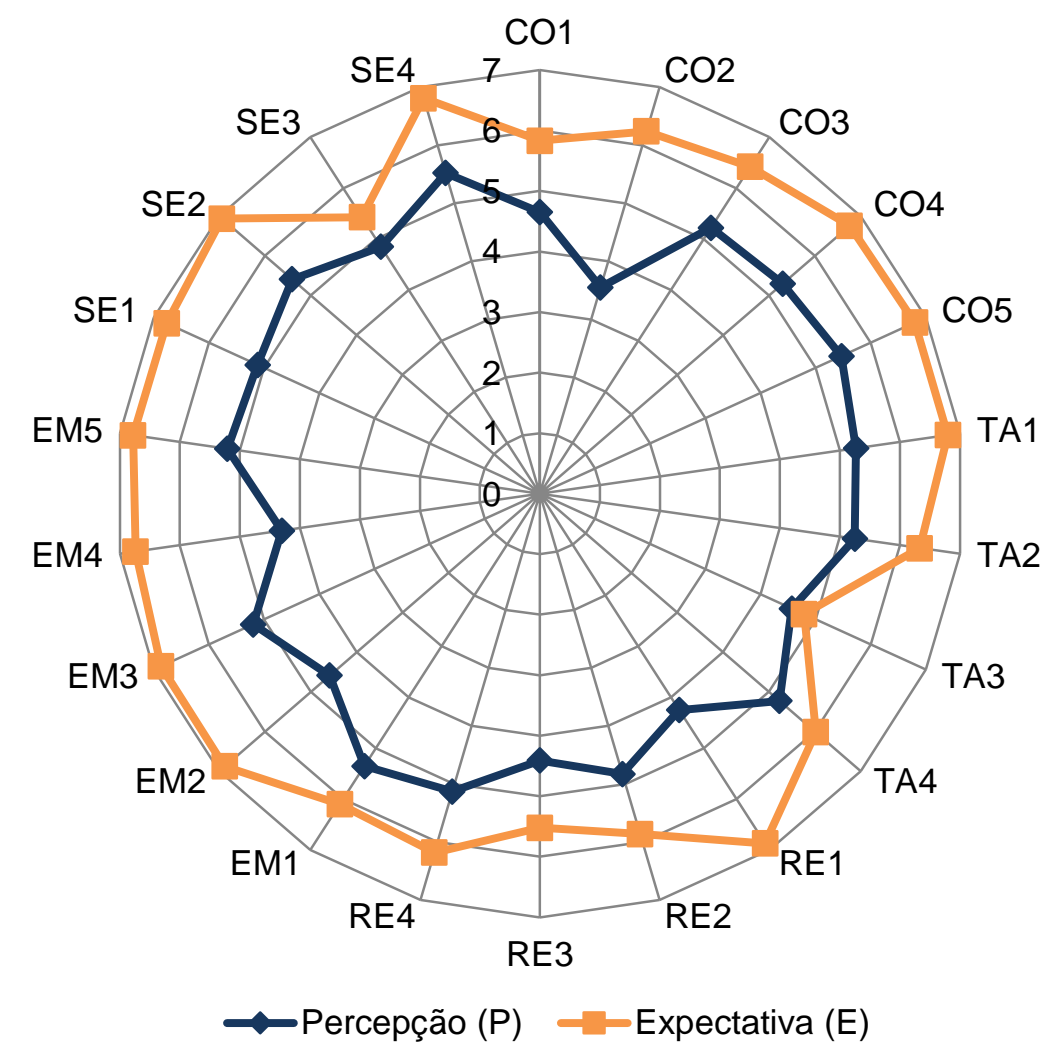

Fonte: elaborado pelos autores.

Para corroborar com as análises apresentadas, foi realizada a análise de quartis (Tabela 5). Os quartis dividem um conjunto de dados em quatro partes iguais, de modo a identificar as variáveis com prioridade crítica, alta, moderada e baixa. O primeiro quartil representa um conjunto dos $25 \%$ menores valores. $O$ segundo quartil pode é propriamente a mediana do conjunto, e separa os $50 \%$ dos menores valores dos $50 \%$ maiores. O terceiro quartil é o valor que delimita os $25 \%$ maiores valores do conjunto. Neste estudo, os quartis foram encontrados por meio do software Statistical Package for the Social Sciences (SPSS ${ }^{\circledR}$ ).

Como pode ser visualizado, no que concerne à expectativa, os itens SE4, RE1, EM3, EM2 e SE2 foram considerados os mais críticos, em consequência de que os consumidores atribuem maior expectativa. Dessa maneira, empresas responsáveis pelo transporte de passageiros por aplicativos devem preocupar-se com aspectos relacionados à imagem e reputação, avisos sobre cancelamentos e atrasos do serviço, atendimento cortês e igualitário aos passageiros, esclarecimento claro sobre a política de atrasos e cancelamentos, e segurança operacional. 
Em relação aos resultados referentes às percepções, os itens CO2, RE1, EM4, RE3, TA3 e EM2 foram considerados críticos em virtude da avaliação negativa dada pelos consumidores. Assim, pode-se considerar que os passageiros encontram-se mais insatisfeitos com aspectos relacionados à conferência dos cintos de segurança por parte dos motoristas, avisos sobre cancelamentos e atrasos do serviço, adequação ao atendimento de passageiros em condições especiais, resolução rápida de eventuais problemas com o aplicativo, modernização dos veículos, e clareza sobre a política de atrasos e cancelamentos.

Finalmente, ao observar os valores referentes às lacunas entre as percepções e as expectativas dos consumidores, seis itens podem ser considerados críticos por representarem as maiores lacunas encontradas. Os aspectos relacionados à conferência dos cintos de segurança por parte dos motoristas (CO2), avisos sobre cancelamentos e atrasos do serviço (RE1), adequação ao atendimento de passageiros em condições especiais (EM4), clareza sobre a política de atrasos e cancelamentos (EM2), atendimento cortês e igualitário aos passageiros (EM3), e a inspiração de confiança proveniente do comportamento dos motoristas (SE1) são os que mais desapontam ao que os consumidores esperam. Portanto, o preenchimento das lacunas apresentadas por este estudo, especialmente dos elementos críticos, pode ser de grande utilidade para as empresas prestadoras de serviço de transporte de passageiros por aplicativos que almejam a melhoraria da qualidade em seus serviços.

Tabela 5 - Resultados da análise dos quartis

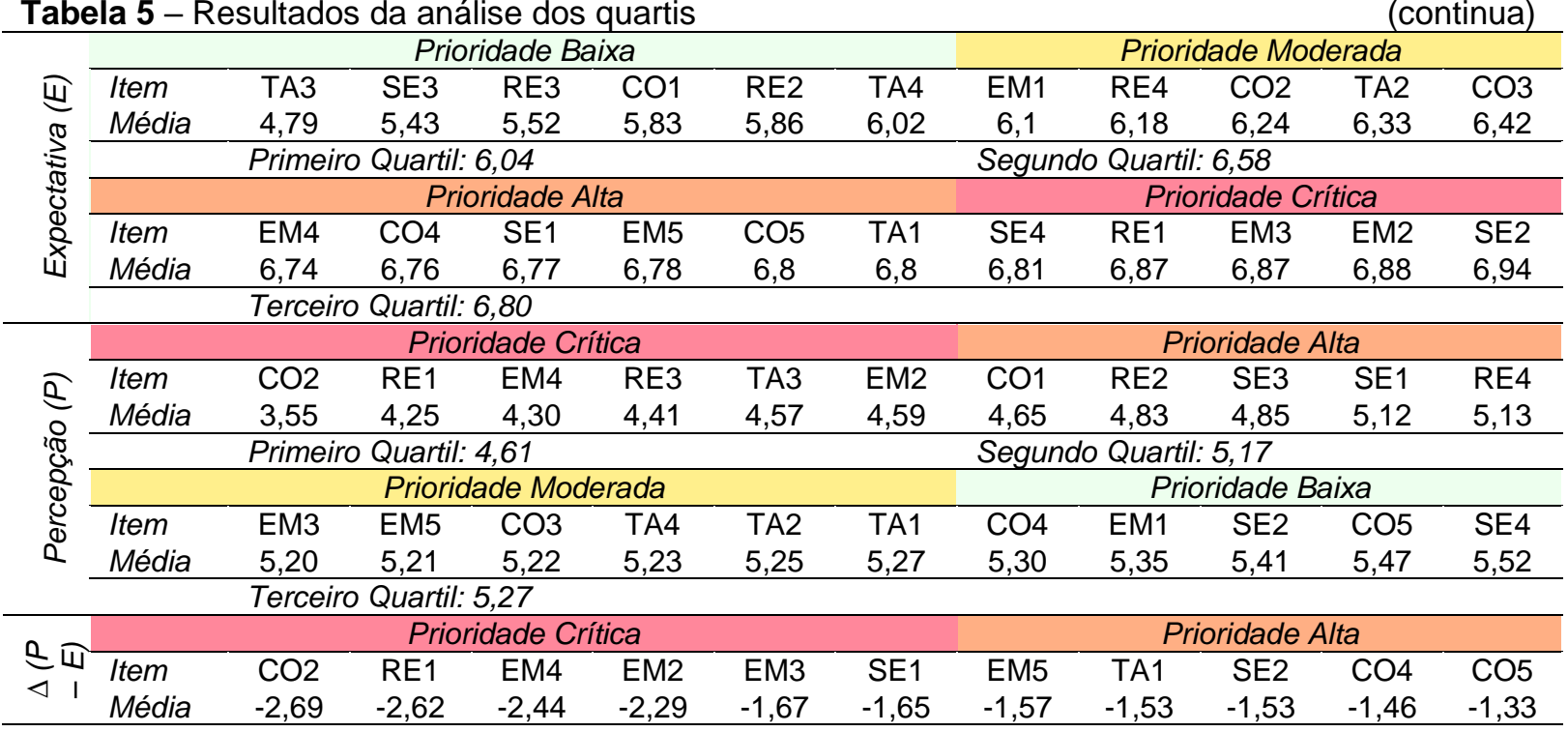


Tabela 5 - Resultados da análise dos quartis

Primeiro Quartil: -1,63

\begin{tabular}{|c|c|c|c|c|c|c|c|c|c|c|c|}
\hline \multicolumn{5}{|c|}{ Primeiro Quartil: -1,63 } & \multicolumn{7}{|c|}{ Segundo Quartil: -1,31 } \\
\hline & & oridad & lodera & & & & & Priorio & Baix & & \\
\hline Item & SE4 & $\mathrm{CO} 3$ & $\mathrm{CO} 1$ & RE3 & TA2 & RE4 & RE2 & TA4 & EM1 & SE3 & TA3 \\
\hline Média & $-1,29$ & $-1,20$ & $-1,18$ & $-1,11$ & $-1,08$ & $-1,05$ & $-1,03$ & $-0,79$ & $-0,75$ & $-0,58$ & $-0,22$ \\
\hline
\end{tabular}

Fonte: elaborado pelos autores.

\section{CONCLUSÃo}

Este trabalho investigou 22 itens que avaliaram a percepção dos consumidores do serviço de transporte de passageiros por aplicativo. Os resultaram demonstraram que os 22 itens possuem lacunas negativas de qualidade de serviço, o que indica que ainda existem de oportunidades de melhoria em todos os atributos do serviço avaliado. Os aspectos que se destacaram por apresentar a maior lacuna entre as expectativas dos consumidores e o desempenho real do serviço prestado foram: conferência do uso de cintos de segurança (CO2); comunicação do motorista ao passageiro sobre o cancelamento ou atraso do serviço (RE1); clareza do aplicativo no esclarecimento sobre a política de atrasos e cancelamentos (EM2);e adequação do serviço no atendimento aos passageiros em condições especiais (EM4).

Do ponto de vista da gestão de serviços, esta pesquisa contribuiu para a compreensão de como os clientes avaliam a qualidade dos serviços de transporte privado por aplicativo e pode ajudar as empresas na concepção e definição de qual experiência desejam proporcionar em sua prestação de serviço, na concepção de seu preço e de suas promoções, entre outros aspectos. Assim, torna-se mais fácil atender aos requisitos exigidos pelos clientes e diferenciar-se dos demais aplicativos disponíveis no mercado, sobressaindo-se no quesito de qualidade do serviço.

Entre todos os atributos mencionados, alguns podem ser associados diretamente à gestão dos aplicativos, enquanto outros dependem diretamente dos motoristas ou de ambos. Os atributos que dependem diretamente da gestão do aplicativo podem ser reavaliados e estruturados para haja uma melhor gestão, seja com a criação de políticas ou condutas mais específicas para os motoristas, ou com um processo mais criterioso de credenciamento de novos motoristas parceiros.

Em relação aos atributos que dependem diretamente do motorista, entendese que os mesmos deveriam proativamente preocupar-se com eles, já que afetam a reputação gerada nos aplicativos e, consequentemente, a continuidade das suas Revista Produção Online. Florianópolis, SC, v. 20, n. 4, p. 1384-1404, 2020. 
atividades. Os atributos dependentes dos motoristas juntamente com o aplicativo podem, de alguma forma, ser controlados a partir de treinamentos ou certificações, proporcionados pela própria empresa para manter um padrão de serviço. Além disso, poderia haver uma fiscalização periodicamente que possibilitasse verificar qual o padrão do serviço que está sendo prestado.

Cabe destacar, no entanto, que os resultados obtidos não podem ser generalizados devido ao método e suas limitações de amostragem. Assim, torna-se relevante a realização de pesquisas futuras que tornem a investigação mais abrangente, com uma amostra mais significativa da população, de tal forma a indicar outros possíveis constructos e atributos que se fizerem relevantes para a avaliação desses serviços.

\section{REFERÊNCIAS}

ABDULLAH, F. Measuring service quality in higher education: HEdPERF versus SERVPERF. Marketing Intelligence \& Planning, v. 24, n. 1, p. 31-47, 2006. https://doi.org/10.1108/02634500610641543

AKDERE, M.; TOP, M.; TEKINGÜNDÜZ, S. Examining patient perceptions of service quality in Turkish hospitals: The SERVPERF model. Total Quality Management \& Business Excellence, p. 1-11, 2018. https://doi.org/10.1080/14783363.2018.1427501

BISHOP, P. A.; HERRON, R. L. Use and misuse of the likert item responses and other ordinal measures. International Journal of Exercise Science, v. 8, n. 3, p. 297, 2015. https://doi.org/10.1108/09684880910951381

BROCHADO, A. Comparing alternative instruments to measure service quality in higher education. Quality Assurance in Education, v. 17, n. 2, p. 174-190, 2009.

COELHO, L. A. DE A. et al. Perfil Socioeconômico dos usuários da Uber e fatores relevantes que influenciam a avaliação desse serviço no Brasil. Congresso Nacional de Pesquisa em Transporte da ANPET. Anais [...], 2017

CONFEDERAÇÃO NACIONAL DE DIRIGENTES LOGISTAS (CNDL) E SERVIÇO DE PROTEÇÃO AO CRÉDITO (SPC) BRASIL. Serviços de transporte por aplicativos. 2017. Disponível em: https://www.spcbrasil.org.br/wpimprensa/wpcontent/uploads/2017/12/An\%25C3\%25A1lise-Mobilidade-Urbana-noBrasil.pdf+\&cd=4\&hl=pt-BR\&ct=clnk\&gl=br. Acesso em: 08 Ago.2019

CRONIN JR, J. J.; TAYLOR, S. A. SERVPERF versus SERVQUAL: reconciling performance-based and perceptions-minus-expectations measurement of service quality. Journal of Marketing, v. 58, n. 1, p. 125-131, 1994.

https://doi.org/10.1177/002224299405800110 
DE BARROS, A. C. P. Uber: o consumo colaborativo e as lógicas do mercado. In: CONGRESSO INTERNACIONAL DE COMUNICAÇÃO E CONSUMO. Anais [...]. 2015.

GHOBADIAN, A.; SPELLER, S.; JONES, M. Service quality: concepts and models. International Journal of Quality \& Reliability Management, v. 11, n. 9, p. 43-66, 1994. https://doi.org/10.1108/02656719410074297

HAIR, J. F. et al., 1998. Multvariate data analysis (5th ed.). New Jersey; Prentice Hall.

HALL, J. V.; KRUEGER, A. B. An analysis of the labor market for Uber's driverpartners in the United States. ILR Review, v. 71, n. 3, p. 705-732, 2018. https://doi.org/10.1177/0019793917717222

HAMING, M. et al. The Application of SERVQUAL Distribution In Measuring Customer Satisfaction of Retails Company. 유통과학연구, v. 17, p. 25-34, 2019. https://doi.org/10.15722/jds.17.2.201902.25

JOIA, L. A.; ALTIERI, D. Adoption of E-Hailing Apps in Brazil : The Passengers Standpoint Full Paper. Twenty-third Americas Conference on Information Systems. Anais [...].2017.

MARÔCCO, A. P. et al. A tecnologia disruptiva presente em aplicativos. Revista Cereus, v. 6, n. 1, p. 125-142, 2004.

NEWMAN, K. Interrogating SERVQUAL: a critical assessment of service quality measurement in a high street retail bank. International Journal of Bank Marketing, v. 19, n. 3, p. 126-139, 2001. https://doi.org/10.1108/02652320110388559

PARASURAMAN, A.; ZEITHAML, V. A.; BERRY, L. L. A conceptual model of service quality and its implications for future research. Journal of Marketing, v. 49, n. 4, p. 41-50, 1985. https://doi.org/10.1177/002224298504900403

PARASURAMAN, A.; ZEITHAML, V. A.; BERRY, L. L. A multiple-item scale for measuring consumer perceptions of service quality. Journal of Retailing, v. 64, n. 1, p. $12-40,1988$.

PARASURAMAN, A.; ZEITHAML, V. A.; BERRY, L. L. Reassessment of expectations as a comparison standard in measuring service quality: implications for further research. Journal of Marketing, v. 58, n. 1, p. 111-124, 1994.

https://doi.org/10.1177/002224299405800109

PRAKASH, G. Understanding service quality: insights from the literature. Journal of Advances in Management Research, v. 16, n. 1, p. 64-90, 2019. https://doi.org/10.1108/JAMR-01-2018-0008

RAMEZ, W. S. Patients' perception of health care quality, satisfaction and behavioral intention: an empirical study in Bahrain. International Journal of Business and

Revista Produção Online. Florianópolis, SC, v. 20, n. 4, p. 1384-1404, 2020. 
Social Science, v. 3, n. 18, 2012.

RANDHEER, K.; AL-MOTAWA, A. A.; VIJAY, P. J. Measuring commuters' perception on service quality using SERVQUAL in public transportation. International Journal of Marketing Studies, v. 3, n. 1, p. 21, 2011. https://doi.org/10.5539/ijms.v3n1p21

RAVICHANDRAN, K. et al. Influence of service quality on customer satisfaction application of SERVQUAL model. International Journal of Business and Management, v. 5, n. 4, p. 117, 2010. https://doi.org/10.5539/ijbm.v5n4p117

RODRIGUES, A. C. M. Uma escala de mensuração da zona de tolerância de consumidores de serviços. Revista de Administração Contemporânea, v. 5, n. 2, p. 113-134, 2001. https://doi.org/10.1590/S1415-65552001000200006

SALAZAR, A.; COSTA, J.; RITA, P. A service quality evaluation scale for the hospitality sector: Dimensions, attributes and behavioural intentions. Worldwide Hospitality and Tourism Themes, v. 2, n. 4, p. 383-397, 2010. https://doi.org/10.1108/17554211011074047

SAM, E. F.; HAMIDU, O.; DANIELS, S. SERVQUAL analysis of public bus transport services in Kumasi metropolis, Ghana: Core user perspectives. Case Studies on Transport Policy, v. 6, n. 1, p. 25-31, 2018. https://doi.org/10.1016/..cstp.2017.12.004

SANTANA, T. A. A. et al. Análise do desempenho das companhias aéreas brasileiras através da escala SERVQUAL. Revista GEPROS, v. 13, n. 1, p. 105, 2018.

SILVA et al. Avaliação da qualidade em serviços: uma análise com relação à percepção dos discentes de uma instituição de ensino superior. Produção OnIne, v. 19, n. 2, p. 722-758, 2019. https://doi.org/10.14488/1676-1901.v19i2.3264

TEODORO, M. C. M.; DA SILVA, Thais Claudia D.'Afonseca; ANTONIETA, Maria. Disrupção, economia compartilhada e o fenômeno Uber. Revista da Faculdade Mineira de Direito, v. 20, n. 39, p. 1-30, 2017. https://doi.org/10.22456/0104-6594.71042

TONGLET, M.; PHILLIPS, P. S.; READ, A. D. Using the Theory of Planned Behaviour to investigate the determinants of recycling behaviour: a case study from Brixworth, UK. Resources, Conservation and Recycling, v. 41, n. 3, p. 191-214, 2004. https://doi.org/10.1016/i.resconrec.2003.11.001

UNUVAR, S.; KAYA, M. Measuring service quality by servperf method: a research on hospitality enterprises. Australian Academy of Accounting and Finance Review, v. 2, n. 4, p. 354-362, 2017.

YUSOFF, M.; MCLEAY, F.; WOODRUFFE-BURTON, H. Dimensions driving business student satisfaction in higher education. Quality Assurance in Education, v. 23, n. 1, p. 86-104, 2015. https://doi.org/10.1108/QAE-08-2013-0035

ZHANG, Y. et al. Which One is More Attractive to Traveler, Taxi or Tailored Taxi? An

Revista Produção Online. Florianópolis, SC, v. 20, n. 4, p. 1384-1404, 2020. 
Empirical Study in China. Procedia Engineering, v. 137, p. 867-875, 2016. https://doi.org/10.1016/j.proeng.2016.01.327

$$
\text { (9) (1) }
$$

Artigo recebido em: 22/09/2020 e aceito para publicação em: 11/12/2020

DOI: http://dx.doi.org/10.14488/1676-1901. v20i4.4132 